EOMmUn: Communication et organisation

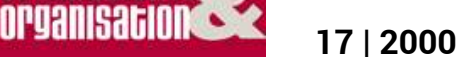

Europe et communication

\title{
Pour une approche communicationnelle de la qualité
}

Valérie Carayol

\section{(2) OpenEdition}

1 Journals

Édition électronique

URL : http://journals.openedition.org/communicationorganisation/2373

DOI : 10.4000/communicationorganisation.2373

ISSN : $1775-3546$

Éditeur

Presses universitaires de Bordeaux

Édition imprimée

Date de publication : 1 mai 2000

ISSN : 1168-5549

Référence électronique

Valérie Carayol, «Pour une approche communicationnelle de la qualité », Communication et

organisation [En ligne], 17 | 2000, mis en ligne le 27 mars 2012, consulté le 19 avril 2019. URL : http://

journals.openedition.org/communicationorganisation/2373; DOI : 10.4000/

communicationorganisation. 2373

Ce document a été généré automatiquement le 19 avril 2019

(c) Presses universitaires de Bordeaux 


\title{
Pour une approche communicationnelle de la qualité
}

\author{
Valérie Carayol
}

1 Nous tenterons de montrer que la conception de la communication qui sous-tend les approches Qualité, qui se focalise sur la circulation de flux à optimiser, les notions de régulation et de contrôle et la chasse à l'aléas, projette une vision de la communication « machinique » sur un univers social. De ce point de vue les démarches Qualité renvoient directement, au regard de la communication, à la première Cybernétique.

2 La perspective que nous allons développer s'appuie sur les écrits d'Edgar Morin et sa critique de la première Cybernétique, développée dans son ouvrage La Méthode (Morin, 1977) et sur les écrits sur la coordination d'Henry Mintzberg (Mintzberg, 1990) issus de son ouvrage Le management, voyage au centre des organisations. A partir de leur lecture et d'une analyse personnelle étayée par des observations empiriques, elle apportera des éléments de réflexion sur les limites des méthodologies Qualité dans les projets complexes.

\section{Edgar Morin et la critique de la Cybernétique}

3 On ne reprendra pas ici, bien entendu, un exposé général sur la Cybernétique, mais on en dira seulement quelques mots, utiles à la compréhension du contexte de l'analyse.

4 La théorie Cybernétique fondée entre 1942 et 1948 par Norbert Wiener (Wiener, 1952) a apporté de nombreux concepts permettant d'analyser les organisations - au sens large fondées sur la communication, et principalement les automates ou machines artificielles dites hétéronomes, soit dépendant de leur environnement. Elle a, selon Edgar Morin, (Morin, 1977, p 254) réunit en un paradigme les deux concepts de communication et de commande (ou régulation) en subordonnant la communication à la régulation. C'est notamment à la Cybernétique que l'on doit les concepts aujourd'hui bien connus de "flux », et de " rétroaction », ainsi que les notions « d'entrée/sortie », de «boite noire » et d'« homéostasie». 
5 Les nombreuses extensions des concepts cybernétiques à la sphère biologique ou sociale ont considérablement étendu leurs applications. Les concepts ont été exportés et appliqués aux systèmes vivants et sociétaux avec plus ou moins de bonheur. Edgar Morin a entrepris une critique de cette première Cybernétique ${ }^{1}$ et des usages abusifs de ses concepts dans un texte incisif dont nous livrons une partie ici (Morin, 1977, pp 252-253) :

6 «Il est né de la cybernétique, une vulgate cybernétoïde, où les termes de rétroaction et d'information, devenus maîtres mots, au lieu d'exprimer leur complexité profonde, banalisent les mystères de la nature et les problèmes de la culture. Cette vulgate associe en elle le réductionnisme engeeneral (sic) et l'impérialisme pan-cybernétique. Elle conçoit la vie selon les fonctionnalités informatiques de la machine artificielle. Ainsi les assauts de cette vulgate sur l'être vivant et l'être social ont pu justement être perçus comme un des aspects du formidable expansionnisme tous azimut de la pensée technocratique, comme une nouvelle forme industrialisée du réductionnisme qui ramène toujours le complexe au simple (ici la réduction de l'organisation vivante aux principes organisationnels de la machine artificielle), comme une réoffensive du machinisme cartésien, qui, cette fois, non content de se borner à réinvestir l'animal, s'efforce d'annexer l'homme et la société.

7 [...] Nous l'avons déjà vu ici même: le modèle de l'artefact cybernétique, projeté sur la société, est le modèle de l'asservissement intégral, parce qu'intégré. [...] En effet, une telle cybernétique, étendant la vision d'un ingénieur sur machines à toute la vaste sphère anthropo-sociale, tend et prétend naturellement à tout réduire à son modèle de soi-disant rationalité : la machine automatisée, fonctionnalisée, purgée de tous désordres (soi-disant optimisée), finalisée pour la production industrielle. Elle ne peut considérer la société que comme une vaste machine à fonctionnaliser. »

8 La critique est rude; l'extension des concepts de la première cybernétique à l'univers social favoriserait une forme industrialisée du réductionnisme; en se focalisant notamment sur les concepts de régulation et de feedback, on érigerait l'asservissement en méthode et on transformerait les organisations sociales en vastes machines fonctionnalisées et purgées de tout désordre.

9 Nous allons essayer de montrer que les politiques et démarches Qualité reposent sur une conception de l'organisation et de la communication directement inspirée par la première Cybernétique. Elles envisagent l'organisation comme un « artefact « et reposent sur une vision de la communication «machinique » Elles s'intéressent essentiellement à l'information et la considèrent comme un instrument de régulation, visant à éliminer le désordre et l'aléas, pour atteindre la stabilité.

10 Nous considérerons, pour ce faire, quelques concepts et instruments utilisés dans la mise en œuvre des programmes Qualité, décrits dans des manuels, des supports internes, ou la presse professionnelle. Nous témoignerons ensuite d'une situation rencontrée dans une grande entreprise industrielle utilisant une méthode de conduite de projet -dans le cadre d'un programme Qualité- pour la conduite d'un projet d'innovation; Nous tenterons alors de voir, si les dangers stigmatisés par Edgar Morin dans son texte sur l'usage et le transfert des concepts de la première Cybernétique vers les organisations vivantes ou sociales ont une réalité ou pas. 


\section{Flugramme et logique client-fournisseur}

11 Les démarches Qualité s'appuient sur des outils dont notamment le «flugramme » (Gogue, 1993, p 20), «qui représente graphiquement les relations fonctionnelles de l'entreprise, tout comme l'organigramme représente les relations hiérarchiques ».

Directement lié à la cybernétique, et exploité par le courant systémique, le flugramme représente l'entreprise comme un système "entrée-sortie «et se focalise sur des processus de production qui, cumulés, sont censés représenter toutes les fonctions de l'entreprise. Est appelé processus « un enchaînement ordonné d'actions et d'événements aboutissant à un résultat déterminé ». (Gogue, 1993, p 21). L'entreprise est conçue comme un ensemble de processus qui s'enchainent, tels des rouages ou «système dont les variations de sorties dépendent d'un système de causes ». (Gogue, 1993, p64)

13 L'entreprise est donc bien considérée comme un artefact, si l'on se réfère à la définition donnée par Jean Pierre Dupuy de l'artefact: «un artefact finalisé peut en général être décrit comme un système "entrée-sortie " L'entrée est constituée de variables d'environnements et/ou de commande, la sortie par la ou les variables qui caractérisent l'objectif à atteindre. » (Dupuy, 1990, p 113)

14 La logique «client-fournisseur» et la notion de «contrat de service» au cœur des politiques Qualité, illustrent également la logique « entrée-sortie » de la cybernétique et le concept de «boite noire » Le dialogue client/fournisseur est défini comme ayant pour « objectif » d'optimiser la performance globale grâce à la détection, la compréhension et la réduction des problèmes de frontières entre les unités $»^{2}$ On s'intéresse ici aux flux d'entrée et de sortie d'information des unités de production, sans trop s'attarder aux conditions de sélection, de construction ou d'élaboration même de l'information. L'intérêt pour les interactions se concentre sur celles qui existent aux frontières des unités et qui concernent l'information opérationnelle uniquement. Seule l'organisation formelle est considérée : la communication concerne seulement l'optimisation des flux d'information pour assurer « fiabilité » et performance globale.

\section{La régulation par la satisfaction du client}

15 Dans une perspective Qualité, toute l'organisation doit être régulée par la mesure de la satisfaction de la clientèle. La qualité du service rendu au client, mesurée par des baromètres et enquêtes de satisfaction, est l'indicateur de réussite du projet. La rétroaction, le feedback sont donnés par cet indicateur. Rappelons que la rétroaction (en anglais feedback) est définie par Norbert Wiener comme La façon de bien commander un système en l'informant du résultat de son action sur l'extérieur. (Gerardin, 1993)

16 L'entreprise tire sa légitimité de la satisfaction de sa clientèle, comme on peut, le constater à la lecture d'articles tirés du Magazine L'Expression d'Entreprise, portant sur la Qualité parus entre 1995 et 1997 (voir bibliographie). Cette satisfaction, au-delà d'un instrument de régulation, devient également un argument de vente.

17 Si l'amélioration de la productivité et la baisse des coûts sont des arguments qui incitent à mettre en des politiques Qualité, c'est la satisfaction du client qui est généralement évoquée comme l'indicateur décisif de son succès. L'entreprise, pilotée par la demande de la clientèle doit mettre tout en pour la satisfaire. 


\section{Contrôle, évaluation et mesure}

de garantir le niveau de fiabilité recherché. L'état d'esprit Qualité, c'est justement le fait d'accepter l'évaluation, en sachant que l'on peut se tromper et qu'il y a toujours place pour l'amélioration » La mesure de l'écart à la norme est mise en œuvre dans un souci de respect de l'organisation formalisée et d'acceptation. L'introduction des méthodes quantitatives dans la mesure de l'activité administrative introduit la culture de la mesure, jusque-là limitée à la production, dans tous les services de l'entreprise. Le travail administratif, considéré comme une suite de processus, peut être évalué et contrôlé, modélisé et calibré. On a bien ici extension de la logique des ingénieurs sur machine à toute l'entreprise. La perte d'autonomie entraînée par ces transformations, bien étudiée en ce qui concerne les systèmes experts et les systèmes interactifs d'aide à la décision a été décrite comme susceptible de créer des situations de rejet, et de perte de motivation (Pomerol, 1990).

\section{La purge de tout désordre ou la chasse au virus de la non-qualité}

Si l'on observe un certain nombre d'écrits sur la Qualité, celle-ci semble effectivement viser la fluidité sociale et la purge de tout désordre. Le mythe de la "commande parfaite », de l'ordre transmis et appliqué sans «bruit » et sans « interférence », du vœu du client exaucé sans "retard», tout comme celui de la transmission parfaite de l'information, sans rumeur et sans erreur permettent d'assimiler, bien souvent, le souci de la Qualité dans les organisations avec celui de la communication. Les services et les 
directions Qualité se sont développées d'ailleurs à la même période que les services communication.

Prenons par exemple une citation de l'ouvrage: Implanter et gérer la qualité totale (Bernillon, Cerutti, 1988, p 23)

«Une entreprise est comme un être vivant; elle ne peut remplir ses fonctions que si elle est en bonne santé. Malheureusement il lui arrive d'être malade. Si l'on examine de plus près ses fonctions (services, bureaux, ateliers, magasins), on y décèle parfois des tensions internes, des conflits sociaux dus à des cloisonnements ou des défauts d'organisation, qui sont autant d'entraves à la communication et à la coopération. Tout cela favorise le développement d'un virus particulièrement insidieux : la non-qualité, dont les conséquences sont actuellement très graves pour l'entreprise. Or si elle souffre de non-qualité, c'est le plus souvent parce que la compétence technique des ingénieurs, des techniciens et le savoir-faire des ouvriers sont trahis par ces cloisonnements et ces défauts d'organisation et de méthode. Ce virus contamine toutes les fonctions et l'entreprise rongée par ses problèmes internes, se referme alors sur elle-même et sur ses dysfonctionnements, en oubliant sa vocation première qui est de satisfaire les besoins de ses clients. »

Les méthodes Qualité sont présentées ici comme un remède, pour poursuivre la métaphore médicale, au désordre, aux cloisonnements, conflits sociaux et autres tensions qui viennent perturber la logique technique des ingénieurs et des techniciens.

Les pratiques participatives qui sont prônées dans les démarches Qualité n'ont pas réellement vocation comme dans les approches Socio-techniques, et les expérimentations issues du courant de la Démocratie Industrielle à favoriser le partage des responsabilités et la gestion en commun de l'organisation ; elles sont plutôt envisagées comme étant des outils au service d'un projet technique ou des moyens efficaces d'aménager un consensus, comme le souligne Michel Crozier (Crozier, 1989, p 67) :

«On croit trop facilement que le consensus est un préalable pour assurer le développement et la rénovation d'une organisation. Et on cherche à le créer en travaillant sur les motivations, c'est à dire l'affectivité du personnel.

L'expérience montre pourtant qu'on n'obtient guère de résultats convaincants quand on prêche des attitudes et des valeurs consensuelles. Le consensus n'est pas un préalable à l'action. Il s'élabore dans l'action. Un cercle de qualité réussi permet d'élaborer un consensus limité, mais réel. »

31 La chasse au désordre est aussi très sensible dans l'énoncé des « cinq zéros olympiques » (Bernillon, Cerutti, 1988, p 28) qui forment une partie des concepts clés de la Qualité : zéro stock, zéro défaut, zéro papier, zéro délai, zéro panne.

Cette chasse au désordre, aux aléas et aux « écarts « détectables avec la multiplication des outils statistiques, correspond à une interprétation de l'ordre, de l'équilibre comme des états souhaitables et nécessaires dans l'entreprise. Cette volonté de stabilité et "d'homéostasie " pour reprendre un terme issu de la Cybernétique souligne, de nouveau, le parallèle entre organisation et machine auto-régulée ou artefact.

33 C'est cette chasse au désordre qui est stigmatisée par Edgar Morin (Morin, 1977, p 252) :

«Il manque fondamentalement à la cybernétique un principe de complexité qui lui permette d'inclure l'idée du désordre. C'est pourquoi elle est incapable de concevoir la réorganisation permanente, l'antagonisme, le conflit, et, par là, incapable de concevoir l'originalité des êtres-machines naturels. Le formalisme cybernétique a le mérite d'unifier sous les mêmes catégories des traits organisationnels propres aux sphères séparées des machines physiques, des machines vivantes, des machines sociales, mais ce formalisme qui désubstantialise fort justement ce qu'il touche, est incapable de concevoir l'être et l'existence. Il lui manque le sens existentiel, 
écologique et organisationnel de l'idée d'ouverture, le sens ontologique de la fermeture (le soi). Il n'y a, ni essence (ce qui est un avantage), ni existence (ce qui est une carence) dans la saisie cybernétique de l'être vivant, ce qui devient très grave dés qu'un cybernétisme prétend interpréter et traiter la vie, l'homme, la société. Ainsi la cybernétique donne un squelette d'organisation au vivant, mais lui retire la vie. Incapable d'introduire la vie dans une machine artificielle, une telle cybernétique est trop capable d'introduire son absence de vie dans nos vies individuelles et notre vie sociale, d'où des conséquences à la fois débilitantes sur le plan théorique et éventuellement terrifiantes sur le plan pratique. »

La chasse au désordre et à l'informel semble également permanente, si l'on interprète les politiques Qualité comme ayant un souci de rationalisation passant essentiellement par la coordination.

\section{La Qualité comme instrument de coordination}

L'importance donnée au concept de fiabilité, de réduction des écarts, par rapport à des normes définies, la focalisation sur la logique "client-fournisseur » soulignent l'intérêt donné dans les programmes Qualité au concept de coordination. Reprenons les différents types de coordination définis par Henry Mintzberg (Mintzberg, 1990) au nombre de six :

- L'ajustement mutuel qui réalise la coordination du travail par le simple processus de la communication informelle.

37 - La supervision directe qui réalise la coordination par le biais d'une seule personne qui donne les ordres et les instructions à plusieurs autres.

- La standardisation des procédés de travail qui réalise la coordination en spécifiant les procédés de travail de ceux qui doivent réaliser des tâches interdépendantes.

39 - La standardisation des résultats qui réalise la coordination en spécifiant les résultats des différents types de travail.

40 - La standardisation des qualifications (et du savoir) qui réalise la coordination par le biais de la formation spécifique de celui qui exécute le travail.

41 - La standardisation des normes, dans lesquelles ce sont les normes qui dictent le travail et qui permettent un référentiel commun aux membres de l'organisation.

42 On s'aperçoit que les modes de coordination privilégiés par les démarches Qualité sont les différents types de standardisation et la normalisation. Ces modes de coordination dictés, pour la standardisation, par la technostructure, si l'on reprend le vocabulaire d'Henry Mintzberg, et, pour les normes, par l'environnement, dépossèdent la ligne hiérarchique et les opérateurs d'une partie de leur pouvoir sur l'activité. Les modes de coordination par ajustement mutuel sont évacués, alors qu'ils sont essentiels, comme le souligne justement Mintzberg (Mintzberg, 1990) aux formes complexes de travail :

«Ces mécanismes de coordination peuvent être considérés comme les éléments les plus fondamentaux de la structure, le ciment qui tient toutes les pierres de la bâtisse de l'organisation. Ils peuvent donner l'impression de se présenter dans un ordre très strict: au fur et à mesure que le travail devient plus compliqué, les moyens favoris de coordination paraissent passer de la forme d'ajustement mutuel (le mécanisme le plus simple) à la supervision directe, puis à la standardisation, de préférence de type des procédés de travail ou des normes, mais aussi des résultats ou des qualifications, pour finalement revenir à celle de l'ajustement mutuel (car paradoxalement, c'est le mécanisme qui est le mieux à même de correspondre à des formes complexes de travail) » 

la lourdeur des procédures. La structure matricielle de l'organisation avait supprimé des niveaux hiérarchiques pour responsabiliser. Les pratiques dans le projet avaient tendance, à l'inverse, à déresponsabiliser. Il y avait moins de délégation de responsabilité au niveau du projet qu'au niveau du travail quotidien pour certains membres, ce qui
faisait parfois passer le projet, pourtant très important stratégiquement, derrière au niveau du projet qu'au niveau du travail quotidien pour certains membres, ce qui
faisait parfois passer le projet, pourtant très important stratégiquement, derrière d'autres activités plus valorisantes pour les agents.

Les besoins en communication fonctionnelle sont réduits au minimum par l'utilisation de procédures; les autres formes de communication et d'interaction plus orientées vers le groupe sont évacuées ou instrumentalisées dans le cas des groupes de discussions, qui sont généralement considérés comme des outils au service d'un projet technique. Il s'agit «d'extraire du quotidien des informations significatives et de les transmettre aux décideurs qui les mettront en œuvre » (Gélinier, 1984).

Limitée à son rôle de coordination, de régulation et de commande, la conception de la communication inscrite dans les projets Qualité est directement inspirée par le projet Cybernétique. De ce point de vue de nombreux effets pervers peuvent être, de notre point de vue, engendrés par la mise en des politiques Qualité.

\section{Les travers de la qualité: une expérience dans une entreprise industrielle à propos d'un projet d'innovation}

L'exemple que je voudrais prendre ici est celui d'une grande entreprise industrielle, à laquelle j'ai été associée dans le cadre d'une équipe de projet d'innovation lourd d'une durée de cinq ans, associant une cinquantaine de personnes.

Cette entreprise utilisait une méthode de développement de projet très élaborée conçue dans le cadre d'une démarche Qualité, à propos de laquelle tout le personnel avait développé une certaine fierté. Fidèle à la tradition industrielle, le processus d'innovation avait été scindé en phases, le tout était très détaillé et la méthodologie de développement du projet, inscrite dans un épais document d'environ une centaine de pages.

L'entreprise, organisée de manière matricielle était à la pointe de toutes les innovations en management et les ingénieurs et techniciens avaient déjà tous été responsabilisés par la participation ou même la conduite de projets de moindre envergure. utilisée, totalement intégrée aux processus qualité. En effet, cette méthode, parfaitement rationnelle d'un point de vue technique venait heurter certains modes d'interaction privilégiés que les acteurs du projet avaient appris à développer ensemble d'une manière informelle et que l'on ne pouvait plus mettre en avec la méthode.

Les individus, habitués à être responsabilisés, se sentaient dépossédés de leur travail par

La culture de l'entreprise, basée sur de nombreuses interactions personnelles informelles se trouvait parfois en décalage avec le formalisme de la méthode. La méthode avait du mal «à prendre» La coordination par les objectifs qui prévalait d'habitude dans l'organisation était remplacée par une coordination de type «procédural «La coordination se faisait par une attribution de tâches et de rôles et par des procédures très définies. Elle rendait les fonctions et les rôles mutuellement exclusifs. Elle ne prévoyait 
pratiquement plus de redondance, pourtant nécessaire à l'ajustement dans une structure d'innovation. De fait, les membres du projet recréaient la redondance là où elle manquait. Ils s'épaulaient, s'aidaient, dans des tâches qui ne relevaient pas de leur service. L'industrialisation, la qualité, le contrôle de gestion aidaient le marketing. Ils créaient des occasions de se voir qui n'étaient pas prévues. L'usage de la méthodologie était parfois transgressé ou détourné, ce qui était une réaction saine de la part de l'organisation. Cette solidarité, cette mise en commun, étaient indispensables à la bonne marche du projet et la réaction d'une organisation saine, capable d'adaptation et souple.

51 Finalement l'organisation a estimé, suivant en cela de nombreuses organisations industrielles, au-delà des aménagements nécessaires à une meilleure prise en compte des besoins en communication interne des membres, de réintroduire de la communication informelle en aménageant un "plateau d'innovation", soit une surface décloisonnée, où les principaux intervenants seraient réunis et auraient des occasions informelles de se voir en se côtoyant.

La rigidification des pratiques provoquée par la multiplication des manuels de procédures qualité, voire des méthodes de conduite de projet, a mis en lumière la nécessité de réintégrer de la communication informelle pour assurer le bon fonctionnement de la structure d'innovation.

\section{Des effets pervers dans les environnements complexes}

53 La mise en garde d'Edgar Morin, relative aux dangers d'une extension des concepts de la première Cybernétique aux univers sociaux nous semble riche et intéressante, même s'il faut évidemment la nuancer. Cette mise en garde est d'autant plus précieuse que, nous l'avons montré, les politiques Qualité reprennent à leur compte bon nombre de concepts et de conceptions issus de la Cybernétique. Quels sont les risques encourus, que l'on peut discerner? On en pointera ici deux.

La focalisation de la Cybernétique sur la "régulation » s'étend aux programmes Qualité. Mais comme le souligne Vincent Defourny (Defourny, 1991, p 53) «La régulation n'est que le cas de maintien des formes stables ou projet et environnement sont permanents. Il faut nécessairement envisager d'autres cas comme l'adaptation, l'apprentissage ou l'innovation qui sont des formes plus complexes d'équilibration ». L'accent mis sur la régulation peut devenir un risque en empêchant la transformation et l'apprentissage. À l'heure où l'entreprise doit réagir en permanence à un environnement changeant, le risque d'une rigidification des pratiques et d'un immobilisme existe. D'une manière générale, la volonté de formalisation à outrance, perceptible dans un certain nombre de programmes Qualité, semble dangereuse pour les situations de travail complexes, pour l'innovation et la créativité. La chasse au désordre, qui devient fréquemment une chasse au temps libre, réduit la redondance des pratiques et les marges de liberté, qui sont, depuis les travaux sur l'innovation, reconnus comme étant indispensable à la créativité. L'inflation de l'activité de certains services Qualité est inquiétante à ce titre. Loin de vouloir organiser pour rendre l'entreprise plus performante, certains services semblent organiser pour organiser.

L'introduction massive de la mesure quantitative induit le risque d'une Retaylorisation de l'entreprise engendrant un rejet de la part des salariés. Les ouvrages analysant les 
pratiques de management les plus critiques, comme par exemple celui de Vincent de Gaulejac et Nicole Aubert Le coût de l'excellence (Gaulejac De, Aubert, 1991) ont montré comment l'entreprise en entrant dans l'ère «managériale « était passée d'une logique de la stimulation des salariés, soumis à un contrôle quantitatif extérieur répressif - ce qu'ils appellent la productivité « dure « - à une logique de l'adhésion ou productivité « douce « . Celle-ci implique, entre autres, plutôt un auto-contrôle des salariés. Dans nombre de projets Qualité, l'auto-contrôle lorsqu'il existe est doublé d'un contrôle extérieur ce qui implique un retour en arrière dans les pratiques de management ou un grand écart pour certaines entreprises entre des pratiques souvent peu compatibles. Ces dissonances peuvent avoir pour conséquence une démotivation et une dé-responsabilisation.

\section{Conclusion}

Dans la littérature organisationnelle, les organisations souples, réactives, matricielles, faisant une grande part à l'autonomie des acteurs de l'organisation sont présentées comme un progrès conséquent par rapport aux structures organisationnelles rigides héritées du taylorisme. Dans le même temps, la démarche Qualité qui, comme nous l'avons montré, considère l'organisation comme un artefact, introduit des pratiques qui viennent heurter les discours et les pratiques de management présentées comme « source d'excellence ». La conception «machinique « de la communication qui les sous-tend, on l'aura montré, est directement issue de la première cybernétique. Discours et pratiques fonctionnent comme des injonctions paradoxales, ou encore comme une «double contrainte " si l'on se réfère à cette notion introduite par Gregory Bateson (Bateson, 1984).

On a le sentiment que toute une partie de la littérature managériale pourtant déjà ancienne est occultée dans les travaux sur la Qualité, notamment les travaux sur l'innovation, les structures organiques et les travaux nés à partir de la deuxième cybernétique. Dés le début des années 1960 on a eu recours aux catégories mécaniques et organiques (Burns, Stalker, 1961) pour décrire la relation dynamique entre la structure et l'environnement. On opposait alors déjà les systèmes mécaniques adaptés aux conditions environnementales stables, possédant une différenciation spécialisée des tâches, des formes de contrôle hiérarchique et une communication utilisant les canaux hiérarchiques verticaux aux systèmes organiques, appropriés aux conditions environnementales changeantes, où les tâches sont susceptibles d'être redéfinies en permanence, des structures de contrôle en réseau impliquant la responsabilisation et une communication distribuée et latérale dans tout le système (Bertrand, Guillemet, 1988, p 82). Les références scientifiques apportées comme des arguments en faveur du développement des pratiques Qualité paraissent, de ce fait, anciennes et désuètes. Car elles ne correspondent pas aux besoins des entreprises complexes et qui évoluent dans des environnements mouvants, qui sont les plus nombreuses aujourd'hui.

D'un point de vue communicationnel, la focalisation de l'attention sur la notion d'information fonctionnelle et sa circulation traduit une méconnaissance de sa nature. Construite et sélectionnée à l'intérieur d'un réseau d'interactions, la qualité de l'information qui est produite par une organisation dépend en grande partie de facteurs humains liés au climat ou au sens attribué au travail. C'est sur la signification attribuée au travail par les salariés qu'il semble urgent de s'interroger. 

choix technologiques, il souligne l'intérêt d'une meilleure compréhension de l'essence et de la finalité du travail, de sa signification pour les équipes et les individus. Cette meilleure compréhension du travail passe selon lui par l'abandon des approches fonctionnelles qui décortiquent analytiquement les processus pour laisser la place à des approches par situation. Celles-ci s'attachent «à identifier la réalité des situations de travail, à comprendre les savoir-faire et leur mode d'acquisition, le lieu et le contexte dans lesquels ils s'inscrivent, les liens entre acteurs, le sentiment de fierté et le sens qui y sont attachés... bref une approche globale, intégrante et non analytique et parcellisante » (André, et alii, 1996, p 191).

61 Si l'on persiste à considérer l'entreprise essentiellement comme une entité de production, le travail comme une suite de processus, et la communication comme un instrument de régulation de l'information fonctionnelle, on ne pourra parler que de production ou coproduction de la Qualité et celle-ci pourra être assimilée à un problème, vaste et compliqué certes, mais un problème de coordination de l'activité.

L'horizon d'une co-construction de la qualité, qui elle, ne nécessiterait plus seulement une coordination mais une véritable coopération dans des environnements complexes, semble encore assez lointain.

63 Nota : Ce texte a fait l'objet, dans une première version, d'une communication au colloque organisé par le LERASS, «Coproduction de la Qualité », Toulouse, 12/13/11/98.

\section{BIBLIOGRAPHIE}

\section{Revues professionnelles}

ARPAGIAN N., « Le groupe Crédit National-BFCE maintient le cap de la qualité totale », in L'Expression d'Entreprise, mai 1997, p. 65.

ARPAGIAN N., « Ford aime les clients heureux et vice-versa », in L'Expression d'Entreprise, février 1997, pp. 73-74.

ARPAGIAN N., « Le GAN souhaite assurer un service de qualité », in L'Expression d'Entreprise, mars 1997, pp. 28-29.

ARPAGIAN N., « Pour Rhône-Poulenc Chimie, communication et qualité vont de pair », in L'Expression d'Entreprise, mars, 1997 pp. 60-61. 
ASPISI A., « Mobiliser sur la qualité », in L'Expression d'Entreprise, août 1995, p. 45.

ASPISI A., « Vivre la qualité » in L'Expression d'Entreprise, mars 1995, pp. 53-54.

DHAM V., «EDF-GDF fixe des normes pour garantir son service », in L'essentiel du Management, mai 1996, pp. 58-68.

DUBOSC J-P., « La BRED place la qualité au service de ses clients », in L'Expression d'Entreprise, mars 1997, pp. 61-62.

DUBOSC J-P., « La SNECMA fait décoller la qualité », in L'Expression d'Entreprise, mars 1997, pp. 28-29.

LAURENT P., « De la qualité dans l'air », in L'Expression d'Entreprise, mai 1995, pp. 21-22.

LAURENT P., « Rank Xerox prépare l'an 2000 », in L'Expression d'Entreprise, avril 1995, p. 32.

LAURENT P., « COGEMA vise l'excellence par la qualité », in L'Expression d'Entreprise, février 1997, p. 31-32.

MARIN M., « La qualité totale selon SODEXHO », in L'Expression d'Entreprise, février, p. 29-30.

ALTER N., Sociologie de l'entreprise et de l'innovation, Paris, PUF, 1996.

ANDRE A. et alii, L'Entreprise digitale. Comment les nouvelles technologies transforment les entreprises françaises, Paris, First Andersen Consulting, 1996.

BALANTZIAN G., L'avantage coopératif. Le partenariat, la coopération, l'alliance stratégique., Paris, Editions d'Organisation, 1997.

BATESON G., La nature et la pensée, Paris, Seuil, 1984.

BERNILLON A., CERUTTI O., Implanter et gérer la qualité totale, Paris, Editions d'Organisation, 1988, col. Management 2000.

BERTRAND Y., GUILLEMET, P., Les organisations, une approche systémique, Paris, Montréal, Ste Foy, Ed Chotard, Agence d'Arc, Télé-Université, 1989.

BLANC G., Le travail au XXI ${ }^{e}$ siècle, Paris, Dunod, 1995.

BRETON P., « Cybernétique », in SFEZ, L., (dir) Dictionnaire de la Communication, PUF, 1993, tome I, pp. 742-744.

BUQUET H., CARAYOL V., SOCHACKI L., Les nouvelles technologies de l'information et de la communication, des outils aux usages, Dossier $n^{\circ} 17$, Renault, Direction de la formation et $\mathrm{du}$ développement social, 129 p., 1998.

BURNS T, STALKER G., The management of innovation, London, Stavistock, 1961.

CARAYOL V., Information et communication interne : les enjeux humains, Journée de l'ANVIE, «Système d'information et performance de l'entreprise : enjeux humains et organisationnels », 8 octobre 1996.

DEFOURNY V., Organisation et pilotage stratégique de la communication, Louvain La Neuve, Faculté des Sciences Economiques, Sociales et Politiques, Université Catholique de Louvain, 1991.

GELINIER O., Stratégie de l'entreprise et motivation des hommes, Paris, Ed. Hommes et Techniques, 1984.

GERARDIN L., Rétroaction, in SFEZ, L., (dir) Dictionnaire de la Communication, PUF, 1993, tome I, pp. 466-467.

GOGUE J.-M., Management de la qualité, Paris, Economica, col. Gestion poche, 1993. 
GOGUE J.-M., Le paradigme de la qualité, Paris, Economica, 1997.

LIVET P., Cybernétique et connexionnisme, in SFEZ, L., (dir) Dictionnaire de la Communication, PUF, 1993, tome I, pp. 208-209.

MINTZBERG H., Le management, voyage au centre des organisations, Paris, Éditions d'Organisation, 1990.

MORIN Edgar, La Méthode. 1- La nature de la nature, Paris, Seuil, 1977.

PAVARD B. (ed.) Systèmes coopératifs : de la modélisation à la conception, Toulouse, Octares, 1994.

PIERRE J., La gestion de la qualité : théories et réalités, in Les Cahiers Français, n² 234, Janv-fév. 1988.

POMEROL J.-C, « Systèmes experts et SIAD : enjeux et conséquences pour les organisations », T.I.S ., vol. 3, n 1, 1990, pp. 44-47.

WIENER, N., Cybernétique et société, Paris, Deux Rives, 1952.

\section{NOTES}

1. On distingue en général la première Cybernétique, celle des créateurs faisant peu de place au désordre, et la seconde Cybernétique ou Cybernétique de second ordre, laissant une place au hasard, à la complexité et aux processus récursifs. Celle-ci a notamment permis d'introduire les concepts d' " auto-organisation « et d'" ordre par le bruit "

2. La Lettre de le Qualité, n 8, février 1993, Caisse Régionale de Crédit Agricole de Charente, p. 4

3. signé Christian Maisonneuve, ingénieur en chef, département qualité

4. La lettre de la qualité, $\mathrm{n}^{\circ}$ 9, octobre 1993, CRCAM de la Charente

\section{RÉSUMÉS}

Cet article introduit une réflexion critique sur les démarches Qualité en montrant que la conception de la communication qui les sous-tend fait largement référence à une vision «machinique » de celle-ci. L'un des objectifs essentiels des méthodes Qualité étant l'optimisation de la coordination, on montrera également que le choix de la standardisation comme procédé principal, tente de faire reculer les méthodes de coordination alternatives, et notamment par ajustement mutuel, en prônant un affaiblissement des communications informelles. Des effets pervers peuvent apparaître dans des contextes où la complexité du travail est grande. Elles peuvent parfois constituer un frein à la créativité et à l'innovation, voire retarder des projets complexes de développement.

This article introduces a critical perspective about quality processes, showing that the underlying conception of communication refers to a "machinic «vision. One of the main objective of Quality processes being the optimisation of coordination, the choice of standardization as the major method try to minimize alternative coordination methods and to weaken informal communications. Perverse effects can emerge in contexts where the complexity of work is important. It may sometimes acts as a break upon creativity and innovation. 
INDEX

Mots-clés : critique, qualité, communication, cybernétique, coordination, innovation, méthodologie de projet

\section{AUTEUR}

VALÉRIE CARAYOL

Valérie Carayol est maître de conférences en sciences de l'information et de la communication à l'Institut des Sciences de l'Information et de la Communication (ISIC) de l'Université Michel de Montaigne, Bordeaux 3. Elle est responsable de la filière communication des organisations de l'IUP et chargée de mission à la communication de l'Université. Chercheur au GREC/O, elle consacre l'essentiel de ses recherches à la communication organisationnelle. 